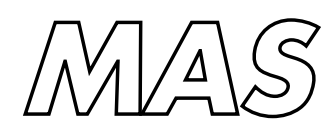

Modelling, Analysis and Simulation

Modelling, Analysis and Simulation
MAS Fourier two-level analysis for discontinuous Galerkin
discretization with linear elements

P.W. Hemker, W. Hoffmann, M.H. van Raalte

Report MAS-R0217 June 30, 2002 
CWI is the National Research Institute for Mathematics and Computer Science. It is sponsored by the Netherlands Organization for Scientific Research (NWO).

$\mathrm{CWI}$ is a founding member of ERCIM, the European Research Consortium for Informatics and Mathematics.

CWI's research has a theme-oriented structure and is grouped into four clusters. Listed below are the names of the clusters and in parentheses their acronyms.

Probability, Networks and Algorithms (PNA)

Software Engineering (SEN)

Modelling, Analysis and Simulation (MAS)

Information Systems (INS)

Copyright @ 2001, Stichting Centrum voor Wiskunde en Informatica

P.O. Box 94079, 1090 GB Amsterdam (NL)

Kruislaan 413, 1098 SJ Amsterdam (NL)

Telephone +31205929333

Telefax +31205924199

ISSN 1386-3703 


\title{
Fourier Two-level Analysis for Discontinuous Galerkin Discretization with Linear Elements
}

\author{
P.W. Hemker,**, W. Hoffmann ${ }^{* *}$ and M.H. van Raalte ${ }^{*, * *}$ \\ *CWI, P.O. Box 94079, 1090 GB Amsterdam, The Netherlands \\ ** KdV Institute for Mathematics, University of Amsterdam \\ Plantage Muidergracht 24, 1018 TV Amsterdam, The Netherlands
}

\begin{abstract}
In this paper we study the convergence of a multigrid method for the solution of a linear second order elliptic equation, discretized by discontinuous Galerkin (DG) methods, and we give a detailed analysis of the convergence for different block-relaxation strategies. In addition to an earlier paper where higher-order methods were studied, here we restrict ourselves to methods using piecewise linear approximations. It is well-known that these methods are unstable if no additional interior penalty is applied.

As for the higher order methods, we find that point-wise block-relaxations give much better results than the classical cell-wise relaxations. Both for the Baumann-Oden and for the symmetric DG method, with a sufficient interior penalty, the block relaxation methods studied (Jacobi, Gauss-Seidel and symmetric GaussSeidel) all make excellent smoothing procedures in a classical multigrid setting. Independent of the mesh size, simple MG cycles give convergence factors $0.2-0.4$ per iteration sweep for the different discretizations studied.
\end{abstract}

2000 Mathematics Subject Classification: 65F10, 65N12, 65N15, 65N30, 65N55

Keywords and Phrases: Discontinuous Galerkin method, multigrid iteration, two-level Fourier analysis, pointwise block-relaxation

\section{INTRODUCTION}

Recently renewed interest arose in discontinuous Galerkin discretizations for partial differential equations of convection diffusion type $[5,6,8]$. An important reason is the new insight in the use of these methods for elliptic equations $[2,3,4]$ and their applicability in $h p$-selfadaptive algorithms [11, 12].

However, thus far relative little attention has been paid to optimally efficient solution methods for the algebraic systems arising from the discretization of the stationary problems. Therefore, we study the possible use of a multigrid algorithm for this purpose. We concentrate on the Baumann DG, the symmetric DG methods [2, 7]. It is well-known $[1,10,13]$ that these methods are not stable for the lowest order of approximation $(p=1)$, if no additional stabilization is applied by means of an interior penalty (IP) parameter. All these methods can 
be described by the same formulas $[2,7]$, where the distinction between the various methods is made by two parameters: $\sigma$, the sign $(\sigma=+1$ for Baumann and $\sigma=-1$ for symmetric $\mathrm{DG})$, and $\mu=\nu / h$, the interior penalty parameter.

Where in an earlier paper [7] we studied the convergence of a multigrid method for the solution of the systems arising from higher order methods $(p \geq 3)$, in the present paper we focus on the convergence of the multigrid method for the case $p=1$. Because this case may be used to accelerate the solution for $p>1$ in the $p$-hierarchical structure of the $h p$-adaptive approximation process.

For the higher order methods we showed that excellent convergence was obtained when blockwise relaxation (Jacobi or Gauss-Seidel) is applied as a smoother, if the blocks are formed by the degrees of freedom (dofs) associated with cell-vertices [7]. This motivates us to study the smoothing abilities for the IP-DG method with a well chosen penalty parameter $\mu$.

The outline of the paper is as follows. In Section 2 we give a unified description of the DG discretizations so that the different symmetric form, Baumann's variant and the Internal Penalty (IP) variants follow from the value of specific parameters $(\sigma$ and $\mu)$ in the formulation. For the linear trial functions that we restrict ourselves to, we give a description of the resulting discrete operator in the form of a stencil that defines the resulting block-Toeplitz matrix.

In Section 3 we apply Fourier analysis to this discrete operator in order to study its stability properties. We observe that both the symmetric DG and Baumann's variant have a double zero eigenvalue, one of which has an eigenfunction that is not constant (the spurious eigenvalue responsible for the instability of the methods). If a large enough penalty parameter is chosen, then it is seen that the instability disappears. However, for too large penalty parameters we see that the discrete system becomes ill-conditioned.

In Section 4 we give a smoothing analysis of the point-wise and cell-wise block-relaxations and a convergence analysis of the two-level algorithm. As in the case for higher degree trial polynomials, dealt with in our earlier paper, we see also here that the use of point-wise relaxation gives much faster convergence. By determining the spectral norm of the erroramplification operator it is shown that the observed "good convergence" is guaranteed from the second iteration step on; we find convergence with a rate of about $0.2-0.4$ per iteration.

In Section 5 we report on numerical results for the solution of a one-dimensional Poisson problem on the unit interval, where the solution has a thin boundary layer, its thickness depending on a parameter $\varepsilon$. The results confirm the theoretical analysis.

\section{The Linear discontinuous Galerkin Discretization}

We consider the Poisson equation on the unit cube $\Omega$, partly with Neumann and partly with Dirichlet boundary conditions:

$$
-\Delta u=f \quad \text { on } \quad \Omega,
$$

with $u=u_{0}$ on $\Gamma_{D}$ and $\mathbf{n} \cdot \nabla u=g$ on $\Gamma_{N}$, where $\Gamma_{D} \cap \Gamma_{N}=\emptyset$ and $\bar{\Gamma}_{D} \cup \bar{\Gamma}_{N}=\partial \Omega$. The variational form of this equation, associated with the DG-methods $[2,7]$ reads: find $u \in H^{1}\left(\Omega_{h}\right)$ such that:

$$
B(u, v)=L(v) \quad \forall v \in H^{1}\left(\Omega_{h}\right),
$$


where

$$
\begin{aligned}
B(u, v) & =\sum_{\Omega_{e} \in \Omega_{h}} \int_{\Omega_{e}} \nabla u \cdot \nabla v d x-\int_{\Gamma_{\mathrm{int}} \cup \Gamma_{\mathrm{D}}}\langle\nabla u\rangle \cdot[v] d s \\
& +\sigma \int_{\Gamma_{\mathrm{int}} \cup \Gamma_{\mathrm{D}}}\langle\nabla v\rangle \cdot[u] d s+\mu \int_{\Gamma_{\mathrm{int}} \cup \Gamma_{\mathrm{D}}}[u] \cdot[v] d s
\end{aligned}
$$

and

$$
L(v)=\sum_{\Omega_{e} \in \Omega_{h}} \int_{\Omega_{e}} f v d x+\sigma \int_{\Gamma_{\mathrm{D}}}\langle\nabla v\rangle \cdot\left[u_{0}\right] d s+\int_{\Gamma_{N}} g v d s .
$$

Here, for non-negative integer $k$, the space $H^{k}\left(\Omega_{h}\right)$ is the broken Sobolev space [3, 9] on the partitioning $\Omega_{h}$ of the domain $\Omega$,

$$
\Omega_{h}=\left\{\Omega_{e} \mid \cup_{e} \bar{\Omega}_{e}=\bar{\Omega}, \Omega_{i} \cap \Omega_{j}=\emptyset, i \neq j\right\} .
$$

The parameter $\mu$ denotes the interior penalty, and $\sigma$ the character of the discretization: $\sigma=1$ gives Baumann's method (or NIPG if $\mu>0$ ), $\sigma=-1$ gives the symmetric DG (IP-DG for $\mu>0)$. The jump operator $[\cdot]$ and the average operator $\langle\cdot\rangle$ are defined at the common interface $^{1}$ between two cells $\Gamma_{i, j}=\bar{\Omega}_{i} \cup \bar{\Omega}_{j}$, by

$$
\begin{aligned}
{[w(x)] } & =\left.w(x)\right|_{\partial \Omega_{i}} \mathbf{n}_{i}+\left.w(x)\right|_{\partial \Omega_{j}} \mathbf{n}_{j}, \\
\langle w(x)\rangle & =\frac{1}{2}\left(\left.w(x)\right|_{\partial \Omega_{i}}+\left.w(x)\right|_{\partial \Omega_{j}}\right),
\end{aligned}
$$

for $x \in \Gamma_{i, j}$. Here $\mathbf{n}_{i}$ is the unit outward pointing normal for cell $\Omega_{i}$. In case of a vector valued function, $\tau$, we define

$$
\begin{aligned}
& {[\tau(x)]=\left.\tau(x)\right|_{\partial \Omega_{i}} \cdot \mathbf{n}_{i}+\left.\tau(x)\right|_{\partial \Omega_{j}} \cdot \mathbf{n}_{j},} \\
& \langle\tau(x)\rangle=\frac{1}{2}\left(\left.\tau(x)\right|_{\partial \Omega_{i}}+\left.\tau(x)\right|_{\partial \Omega_{j}}\right) .
\end{aligned}
$$

The interior boundaries are denoted by $\Gamma_{\text {int }}=\cup \Gamma_{i, j}$.

In this paper we restrict our analysis to the one-dimensional equation, since this can be considered as an essential building block for the higher dimensional case where we use tensor product polynomials. For test and trial space $S_{h} \subset H^{1}\left(\Omega_{h}\right)$ we use the space of discontinuous piecewise polynomials on the partitioning $\Omega_{h}$. Then the discrete equations read: find $u_{h} \in S_{h}$ such that

$$
B\left(u_{h}, v_{h}\right)=L\left(v_{h}\right) \quad \forall v_{h} \in S_{h} .
$$

\footnotetext{
${ }^{1}$ At a Dirichlet boundary the interface with a virtual (flat, exterior) adjacent cell, containing only the Dirichlet data, is used.
} 
With a basis $\left\{\phi_{i, e}\right\}$ for the space $S_{h}$ this leads to the linear system

$$
\begin{gathered}
\sum_{e=1}^{N} \sum_{i=0}^{1} c_{i, e}\left(\int_{\Omega_{e}} \phi_{i, e}^{\prime}(x) \phi_{j, e}^{\prime}(x) d x-\left.\left\langle\phi_{i, e}^{\prime}(x)\right\rangle \cdot\left[\phi_{j, e}(x)\right]\right|_{\Gamma_{\mathrm{int}} \cup \Gamma_{\mathrm{D}}}+\right. \\
\left.\left.\sigma\left[\phi_{i, e}(x)\right] \cdot\left\langle\phi_{j, e}^{\prime}(x)\right\rangle\right|_{\Gamma_{\mathrm{int}} \cup \Gamma_{\mathrm{D}}}+\left.\mu\left[\phi_{i, e}(x)\right] \cdot\left[\phi_{j, e}(x)\right]\right|_{\Gamma_{\mathrm{int}} \cup \Gamma_{\mathrm{D}}}\right)= \\
\sum_{e=1}^{N} \sum_{i=0}^{1} \int_{\Omega_{e}} f \phi_{j, e}(x) d x+\left.\sigma\left[u_{0}\right] \cdot\left\langle\phi_{j, e}^{\prime}(x)\right\rangle\right|_{\Gamma_{\mathrm{D}}}+\left.g \phi_{j, e}(x)\right|_{\Gamma_{N}},
\end{gathered}
$$

which we briefly denote by $L_{h} u_{h}=f_{h}$. In this paper we restrict ourselves to $S_{h}$ consisting of piecewise linear polynomials on a uniform partitioning for which we use the element basis functions $\phi_{j}(\xi)=\xi^{j}(1-\xi)^{1-j}, j=0,1$, so that we have $2 N$ basis functions $\phi_{j}\left(\left(x-x_{e}\right) / h\right)=$ $\phi_{j, e}(t) ; j=0,1 ; e=1, \cdots, N$. For this basis of piecewise linear polynomials the linear system (2.6) has a $2 \times 2$-block-tridiagonal structure, with the discretization stencil:

$$
\frac{1}{h}\left[\begin{array}{cc|cc|cc}
-\frac{1}{2} & \frac{1-\sigma}{2}-h \mu & \frac{1+\sigma}{2}+h \mu & \frac{-1-\sigma}{2} & \frac{1}{2} \sigma & 0 \\
0 & \frac{1}{2} \sigma & \frac{-1-\sigma}{2} & \frac{1+\sigma}{2}+h \mu & \frac{1-\sigma}{2}-h \mu & -\frac{1}{2}
\end{array}\right],
$$

if the equations (the weighting functions $\phi_{e, j}$ ) and coefficients are ordered cellwise as $\left[c_{e, 0}, c_{e, 1}\right]_{e=1}^{N}$. As we emphasized in [7] we can also order the equations and coefficients pointwise, according to function values at the cell-interfaces, $\left[c_{e-1,1}, c_{e, 0}\right]_{e=2}^{N}$, which leads to the stencil:

$$
\frac{1}{h}\left[\begin{array}{cc|cc|cc}
\frac{1}{2} \sigma & \frac{-1-\sigma}{2} & \frac{1+\sigma}{2}+h \mu & \frac{1-\sigma}{2}-h \mu & -\frac{1}{2} & 0 \\
0 & -\frac{1}{2} & \frac{1-\sigma}{2}-h \mu & \frac{1+\sigma}{2}+h \mu & \frac{-1-\sigma}{2} & \frac{1}{2} \sigma
\end{array}\right] .
$$

Thus, with the possible exception for the equations at the boundaries, the discretization matrix appears to be a block-Toeplitz matrix and is described by the repetition of either stencil (2.7) or stencil (2.8). Both stencils describe one and the same matrix, but the distinction between cell-wise and point-wise blocks materializes as soon as we consider block-relaxation methods.

\section{Fourier Analysis of the Discrete operator $B\left(u_{h}, v_{h}\right)$}

In this section we first introduce the Fourier transform of a block-Toeplitz operator. We describe the spectrum of the discrete operator $L_{h}$ on an infinite domain, and we discuss its stability properties. We notice the difference between this operator for piecewise cubic approximations, as described in [7], and the corresponding operator for piecewise linears. We recognize that for the latter a sufficiently large interior penalty parameter has to be chosen in order to obtain a stable scheme. Then, for the stable schemes, we compute the order of accuracy. 
3.1 Fourier analysis for a block Toeplitz operator $A_{h}$

In [7] we have shown that, for $A_{h}=\left(\mathbf{a}_{m, j}\right) \in \mathbb{R}^{2 \mathbb{Z} \times 2 \mathbb{Z}}$ an infinite block Toeplitz operator, we have the identity

$$
\begin{aligned}
& \sum_{j \in \mathbb{Z}} \mathbf{a}_{m, j} e_{h, \omega}(j h)=\widehat{A}_{h}(\omega) e_{h, \omega}(m h), \quad \text { with } \\
& \widehat{A}_{h}(\omega)=\sum_{j \in \mathbb{Z}} \mathbf{a}_{m, j} e^{i(j-m) h \omega}=\sum_{k \in \mathbb{Z}} \mathbf{a}_{-k} e^{i k h \omega}=\sum_{k \in \mathbb{Z}} \mathbf{a}_{k} e^{-i k h \omega},
\end{aligned}
$$

for all $\omega \in \mathbb{T}_{h} \equiv\left[-\frac{\pi}{h}, \frac{\pi}{h}\right]$. Here $e_{h, \omega}(j h)=e^{i j h \omega}$ is an elementary mode, defined on the regular infinite one-dimensional grid

$$
\mathbb{Z}_{h}=\{j h \mid j \in \mathbb{Z}, h>0\}
$$

Furthermore, with $V_{h}=V_{h}(\omega)$ the matrix of eigenvectors $\mathbf{v}(\omega)$ of $\widehat{A}_{h}(\omega)$, such that:

$$
\widehat{A}_{h}(\omega) V_{h}=V_{h} \Lambda_{h}(\omega)
$$

then, with $\left(V_{h} \otimes e_{h, \omega}\right)(j h)=V_{h}(\omega) e^{i j h \omega}$, we have

$$
A_{h}\left(V_{h} \otimes e_{h, \omega}\right)=\widehat{A}_{h}(\omega)\left(V_{h} \otimes e_{h, \omega}\right)=\left(V_{h} \otimes e_{h, \omega}\right) \Lambda_{h}(\omega) .
$$

Hence, the columns $\mathbf{v}(\omega) e_{h, \omega}(m h)$ of $V_{h} \otimes e_{h, \omega}$ are the eigenvectors of $A_{h}$ and $\Lambda_{h}(\omega)$ is a family of $2 \times 2$ diagonal matrices containing the eigenvalues of $A_{h}$ on its diagonal.

\subsection{Eigenvalues spectra of the discrete operator $L_{h}$}

Now we study the eigenvalue spectra of the discrete operator $L_{h}$ of (i) Baumann's, (ii) the symmetric-, and (iii) the internal penalty (IP) DG-method, all with linear elements. It is well known that in this case Baumann's and the symmetric DG-method are unstable and that an additional penalty parameter $\mu=\nu / h$ (IP DG-method) can be introduced in order to stabilize the discrete operator $L_{h}$. To study the behavior of the three different DG-methods we look at the eigenvalue spectra of $\widehat{L_{h}}(\omega)$, since the eigenvalues and eigenvectors of $\widehat{L_{h}}(\omega)$ correspond with the eigenvalues and eigenvectors of $L_{h}$. Considering the point-wise stencil (2.8) we write for $\widehat{L_{h}}(\omega)$, using $(3.1)$,

$$
\widehat{L_{h}}(\omega)=\frac{1}{2 h}\left(\begin{array}{cc}
1+\sigma+2 \nu-e^{\omega h}+\sigma e^{-\omega h} & 1-\sigma-2 \nu-(1+\sigma) e^{-\omega h} \\
1-\sigma-2 \nu-(1+\sigma) e^{\omega h} & 1+\sigma+2 \nu-e^{-\omega h}+\sigma e^{\omega h}
\end{array}\right) .
$$

The eigenvalues $\lambda_{h}(\omega)$ of $\widehat{L_{h}}(\omega)$ for respectively Baumann's, the symmetric and the IP DGmethod are shown in Table 1 . Note that the same eigenvalues are obtained if the cell-wise stencil (2.7) is used instead of the point-wise stencil (2.8). Only the coefficients of the eigenvectors $\mathbf{v}(\omega) e_{h, \omega}(m h)$ are collected either point-wise $\left(\left[c_{e-1,1}, c_{e, 0}\right]\right)$ or cell-wise $\left(\left[c_{e, 0}, c_{e, 1}\right]\right)$.

If we study the eigenvalues $\lambda_{h}(\omega)$ of $\widehat{L_{h}}(\omega)$ for Baumann's DG-method, we see in Table 1 that they are real and non-negative: $\lambda_{1}(\omega), \lambda_{2}(\omega) \in[0,2 / h]$. Furthermore we see that $\lambda_{2}=0$ for $\omega=0$, which is the eigenvalue corresponding to the constant eigenfunction. This eigenfunction corresponds with the equivalent eigenfunction for the continuous operator and 


\begin{tabular}{|c|c|c|c|}
\hline$\lambda_{h}(\omega)$ & Baum-DG & symm-DG & IP-DG \\
& $\sigma=1, \mu=0$ & $\sigma=-1, \mu=0$ & $\sigma=-1, \mu=\nu / h$ \\
\hline$\lambda_{1}(\omega)$ & $\frac{1+\cos (\omega h)}{h}$ & $\frac{1-\cos (\omega h)}{h}$ & $\frac{\nu-\cos (\omega h)+|\nu-1|}{h}$ \\
\hline$\lambda_{2}(\omega)$ & $\frac{1-\cos (\omega h)}{h}$ & $\frac{-1-\cos (\omega h)}{h}$ & $\frac{\nu-\cos (\omega h)-|\nu-1|}{h}$ \\
\hline
\end{tabular}

Table 1: Eigenvalues of $\widehat{L_{h}}(\omega)$.

is controlled by the boundary conditions. However, we see that there also is an additional zero eigenvalue $\lambda_{1}=0$ for $\omega= \pm \pi / h$, and the corresponding eigenvalue is oscillating piecewise constant. This spurious zero eigenvalue causes the Baumann DG-method to be singular for linear basis functions in test and trial space.

The same oscillating piecewise constant function is an additional eigenfunction, with $\lambda_{2}=$ 0 , for the discrete operator $L_{h}$ for the symmetric DG-method. Furthermore, for this DGmethod we recognize the saddle-point behavior $\lambda_{1}(\omega) \in[0,2 / h], \lambda_{2}(\omega) \in[-2 / h, 0]$.

If we study the eigenvalue spectrum $\lambda_{h}(\omega)$ for the IP DG-method, then we still may choose the penalty parameter $\nu$. If we choose $\nu<0$, the method is stable in the sense that the unique zero eigenvalue corresponds to the constant eigenfunction. However the method is indefinite. If we choose the parameter $0 \leq \nu<1$ the method is indefinite and unstable (since then there is a spurious zero eigenvalue with a corresponding oscillating piecewise constant eigenfunction). For $\nu \geq 1$ the method is stable (the eigenvalues have non-negative sign). On the other hand, for a large parameter $\nu$ the discrete operator is ill-conditioned.

Whereas Baumann's non-symmetric DG-method has real positive eigenvalues for linear polynomials in the test and trial space, this is not the case for higher order piecewise polynomials. Figure 1 shows the eigenvalue spectra the Baumann and the symmetric DG-Method for piecewise cubics, as analyzed in [7]. The spectrum of Baumann's method shows complex eigenvalues; in case of the symmetric DG-method the spectrum is real but indefinite. Notice

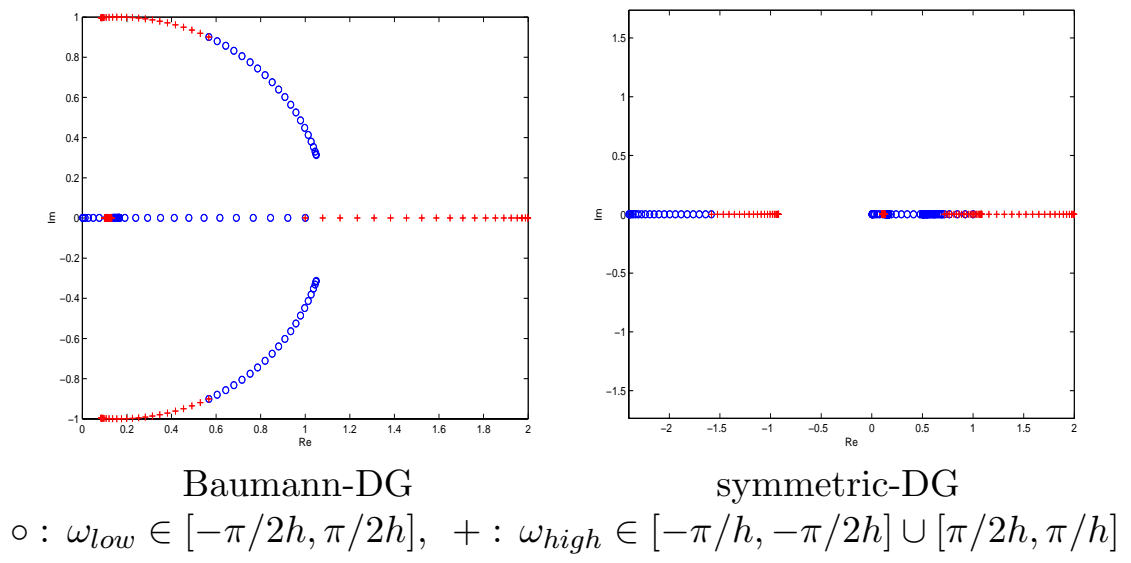

Figure 1: Eigenvalue spectra of discrete operator $\widehat{L_{h}}(\omega)$ for cubic stencil.

the distinction between eigenvalues for low and high frequencies which is useful in the context of multigrid. (Low frequency functions can also be represented on a twice coarser grid.) 


\subsection{Consistency of the IP DG-method}

In the previous section we have seen that the IP DG-method for the piecewise linear basis is stable if $\nu \geq 1$. In this section we study the accuracy and the discrete convergence of this method. For the analysis we use the point-wise stencil (2.8) and analogous to the treatment in [7] we study the truncation operator

$$
\tau_{h}=L_{h} R_{h}-\bar{R}_{h} L,
$$

and the operator corresponding with the discrete convergence, $C_{h}=L_{h}^{-1} \tau_{h}$. In (3.5) $R_{h}$ : $C^{1}\left(\Omega_{h}\right) \rightarrow \mathbb{R}^{2 \mathbb{Z}_{h}}$ is the injective restriction defined by

$$
\mathbf{u}_{h}(j h)=\left(R_{h} u\right)(j h)=\left[\begin{array}{l}
\left.u(j h)\right|_{\Omega_{j-1}} \\
\left.u(j h)\right|_{\Omega_{j}}
\end{array}\right] .
$$

The second restriction, $\bar{R}_{h}: C^{1}\left(\Omega_{h}\right) \rightarrow \mathbb{R}^{2 \mathbb{Z}_{h}}$, is the Galerkin restriction defined by

$$
\left(\bar{R}_{h} f\right)(j h)=\left[\begin{array}{l}
\int_{(j-1) h}^{j h} \phi_{1, j-1}(x) f(x) d x, \\
\int_{j h}^{(j+1) h} \phi_{0, j}(x) f(x) d x,
\end{array}\right.
$$

for all $f \in L^{2}(\Omega)$. Using $\tau_{h} e_{\omega}$ for the truncation error

$$
\tau_{h} e_{\omega}(x)=\tau_{h} e^{i \omega x}=\left(L_{h} R_{h} e_{\omega}-\bar{R}_{h} L e_{\omega}\right)(x),
$$

and with the definition of $R_{h}$ we find

$$
\tau_{h} e_{\omega}=L_{h} e^{i \omega j h}\left[\begin{array}{l}
1 \\
1
\end{array}\right]-\omega^{2} h e^{i \omega j h}\left[\begin{array}{l}
\int_{0}^{1} e^{i \omega h(t-1)} t d t \\
\int_{0}^{1} e^{i \omega h t}(1-t) d t
\end{array}\right],
$$

where the basis functions are scaled to the master element $\widehat{\Omega}=[0,1]$. So,

$$
\begin{aligned}
\tau_{h} e_{\omega} & =\left(\widehat{L_{h}}(\omega)\left[\begin{array}{l}
1 \\
1
\end{array}\right]-h\left[\begin{array}{l}
\int_{0}^{1} e^{i \omega h(t-1)} t d t \\
\int_{0}^{1} e^{i \omega h t}(1-t) d t
\end{array}\right] \omega^{2}\right) e^{i \omega j h} \\
& =:\left(\widehat{L_{h}}(\omega) \widehat{R_{h}}(\omega)-\widehat{\widehat{R}}_{h}(\omega) \widehat{L}(\omega)\right) e^{i \omega j h}
\end{aligned}
$$

where $\widehat{L_{h}}(\omega)$ is the Fourier transform of the block Toeplitz matrix $L_{h}$ for the point-wise stencil. The order of the truncation error is found by expansion of (3.6) for $h \rightarrow 0$. Since $e_{\omega}=e^{i \omega x}$ is continuous, both for Baumann's method $(\sigma=1, \mu=0)$, and for the symmetric DG-method without penalty $(\sigma=-1, \mu=0)$ and with interior penalty $(\sigma=-1, \mu=\nu / h)$, the absolute value of the truncation error is

$$
\left|\tau e_{\omega}\right|=\left[\begin{array}{c}
\frac{1}{6} h^{2} \omega^{3}+O\left(h^{3} \omega^{4}\right) \\
\frac{1}{6} h^{2} \omega^{3}+O\left(h^{3} \omega^{4}\right)
\end{array}\right]
$$

However from the previous section we know that only the IP DG-method is stable and definite, provided we choose $\nu \geq 1$. So, for that method we can derive the discrete convergence from

$$
L_{h}^{-1} \tau_{h} e_{\omega}={\widehat{L_{h}}}^{-1}(\omega)\left(\widehat{L_{h}}(\omega) \widehat{R_{h}}(\omega)-\widehat{\bar{R}}_{h}(\omega) \widehat{L}(\omega)\right) e^{i \omega j h} .
$$

The results are summarized in Table 2 , distinguishing between penalty parameters $\mu=1 / h$ and $\mu=\nu / h$ with $\nu>1$. We see that we loose two orders of accuracy if $\mu=1 / h$. The IP DG-method is more accurate for a larger constant $\nu$, but on the other hand, the method becomes less attractive due to the worse condition number of the discrete operator $L_{h}$. 


$$
\begin{array}{|c|c|}
\hline \mathrm{IP}, \mu=1 / h & \mathrm{IP}, \mu=\nu / h, \nu>1 \\
\hline\left(\begin{array}{c}
-\frac{1}{3} h \omega+O\left(h^{2} \omega^{2}\right) \\
+\frac{1}{3} h \omega+O\left(h^{2} \omega^{2}\right)
\end{array}\right) & \left(\begin{array}{c}
-\frac{1}{12(\nu-1)} h^{3} \omega^{3}+O\left(h^{4} \omega^{4}\right) \\
+\frac{1}{12(\nu-1)} h^{3} \omega^{3}+O\left(h^{4} \omega^{4}\right)
\end{array}\right) \\
\hline
\end{array}
$$

Table 2: The expansion of (3.8) for $h \rightarrow 0$, i.e., the order of convergence of pointwise values at the nodal points.

\section{Smoothing Analysis And COnVERGence of the TWO-Level Algorithm}

In this section we consider three block relaxation methods: Jacobi-, Gauss-Seidel-, and symmetric Gauss-Seidel block relaxations. If we want to apply these relaxations to the unstable operators (Baumann or symmetric DG with $\mu=0$ ) with cell-wise blocks, then we notice that (i) it is impossible to apply Jacobi relaxation because of the singular diagonal blocks, and (ii) that block GS doesn't converge because all eigenvalues of the iteration operator have absolute value equal to 1. Point-wise block relaxation can be used. However, as can be expected, spurious modes remain and no smoothing is achieved.

For the stabilized methods, with $\mu \geq 1 / h$, all block relaxations are smoothers, but for $\mu>1 / h$ point-wise block methods perform much better than the cell-wise block equivalents.

Because of this result, later in this section we drop the cell-wise relaxation and analyze two-level convergence for each of the three point-wise block relaxations. We determine the spectrum of the two-level iteration operator (for different values of $\mu$ ) and compute for each of the relaxations the optimal damping parameter and the corresponding convergence rate.

Finally, in order to show that fast convergence is not only an asymptotic property after many iterations, but can be expected already in the first steps, we determine the spectral norms for the iteration operators at the end of Section 4.2.

\subsection{Smoothing analysis}

Having shown in [7] for piecewise cubics that the smoothing properties of the damped blockJacobi (JOR) and the damped block-Gauss Seidel (DGS) are better for point-wise ordering than for cell-wise ordering, we see the same for piecewise linear basis functions. In this section we analyze the different smoothers for the linear case, again distinguishing between the cell-wise (2.7) and point-wise (2.8) approach.

For the discrete system $A_{h} x=b$ we consider the iterative process

$$
x^{(i+1)}=x^{(i)}-B_{h}\left(A_{h} x^{(i)}-b\right),
$$

with $B_{h}$ an approximate inverse of $A_{h}$. Decomposing $A_{h}$ as

$$
A_{h}=L+D+U
$$

into a strictly block-lower, a block-diagonal and a strictly block-upper matrix, the different relaxation methods are uniquely described either by $B_{h}$ or by the amplification matrix $M_{h}^{\mathrm{REL}}=I_{h}-B_{h} A_{h}$. These operators are shown in Table 3 . Because $A_{h}$ is a block Toeplitz operator, also the amplification matrix $M_{h}$ is block Toeplitz. Notice, that the meaning of the block decomposition (4.2) is different for the stencils (2.7) and (2.8). The stencils corresponding with the decomposition (4.2) are given in Table 4. As we emphasized in [7], the difference 


\begin{tabular}{|c|c|c|}
\hline & $B_{h}$ & $M_{h}^{R E L}$ \\
\hline$J O R$ & $\alpha D^{-1}$ & $D^{-1}((1-\alpha) D-\alpha(L+U))$ \\
\hline$D G S_{L}$ & $\alpha(D+L)^{-1}$ & $(D+L)^{-1}((1-\alpha)(D+L)-\alpha U)$ \\
\hline$D G S_{U}$ & $\alpha(D+U)^{-1}$ & $(D+U)^{-1}((1-\alpha)(D+U)-\alpha L)$ \\
\hline \multicolumn{3}{|c|}{$\alpha>0$ is a damping parameter }
\end{tabular}

Table 3: The relaxation methods.

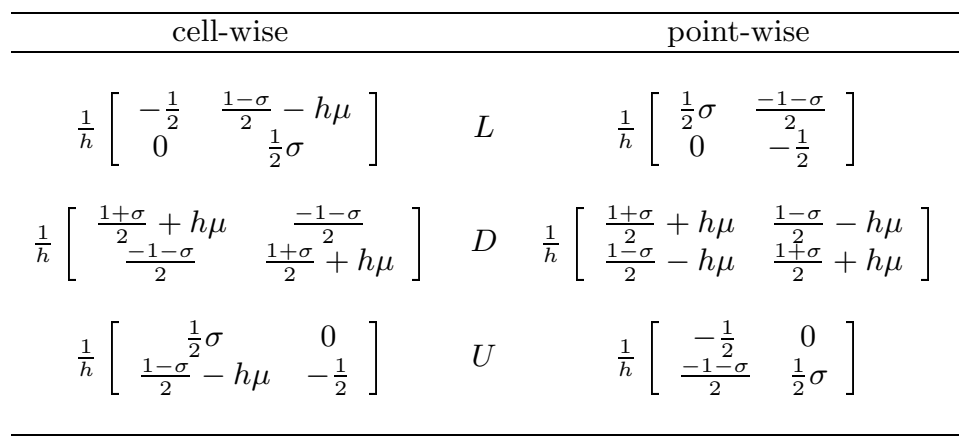

Table 4: The stencils in the diagonal decomposition

between cell-wise and point-wise decomposition is that the eigenvectors $e_{h, \omega}(m h) \mathbf{v}$ of the cellwise stencil correspond with 2-valued grid functions associated with the cell interiors (in fact independently of the basis chosen), whereas for the point-wise stencil they correspond with the 2-valued grid function associated with the nodal points between the cells. This makes the point-wise stencil better suited for a multi-grid algorithm. Using (3.1) we find the Fourier transform of the basic Toeplitz operators: $\widehat{L}(\omega)=L e^{-i \omega h}, \quad \widehat{D}(\omega)=D, \quad \widehat{U}(\omega)=U e^{i \omega h}$. This yields the Fourier transform for the amplification operators for JOR, DGS and SGS:

$$
\begin{aligned}
\widehat{M_{J O R}^{R E L}} & =\widehat{D}^{-1}((1-\alpha) \widehat{D}-\alpha(\widehat{L}+\widehat{U})), \\
\widehat{M_{D G S_{L}}^{R E L}} & =(\widehat{D}+\widehat{L})^{-1}((1-\alpha)(\widehat{D}+\widehat{L})-\alpha \widehat{U}), \\
\widehat{M_{D G S_{U}}^{R E L}} & =(\widehat{D}+\widehat{U})^{-1}((1-\alpha)(\widehat{D}+\widehat{U})-\alpha \widehat{L}), \\
\widehat{M_{S G S}^{R E L}} & =\widehat{M_{D G S_{L}}^{R E L}} \widehat{M_{D G S_{U}}^{R E L}} .
\end{aligned}
$$

By (3.3) we find the eigenvalues of $M_{h}^{R E L}$ by computing the eigenvalues of $\widehat{M_{h}^{R E L}}(\omega)$ for $\omega \in$ $\mathbb{T}_{h}$. The eigenvalues corresponding with the high frequencies $(|\omega|>\pi / 2 h)$, that determine the smoothing properties of the relaxation, are found as $\widehat{M_{h}^{R E L}}(\omega)$ for $\omega \in \mathbb{T}_{h} \backslash \mathbb{T}_{2 h}$. The spectra for the three different smoothers, applied on the DG method of Baumann $(\sigma=1$, $\mu=0)$, the symmetric DG-method $(\sigma=-1, \mu=0)$ and the IP DG-method $(\sigma=-1$, $\mu=\nu / h)$ are shown in the Figures $2-4$ respectively.

The IP DG-method is stable for penalty parameters $\mu=\nu / h, \nu \geq 1$, which is reflected in the fact that the only undamped mode is the constant (eigen) function. 


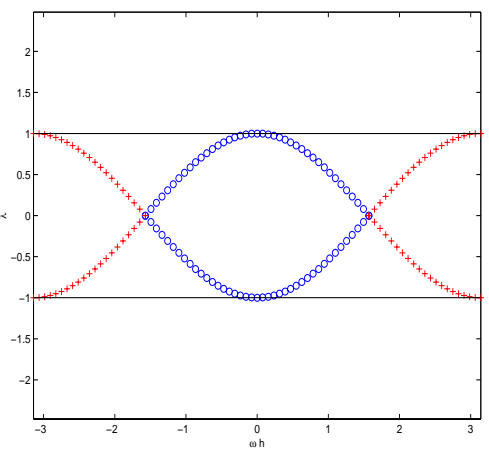

Baumann or symmetric-DG point-wise

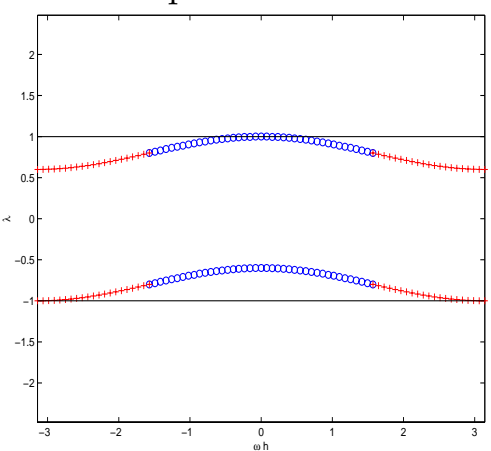

IP-DG $\mu=5 / h$

cell-wise

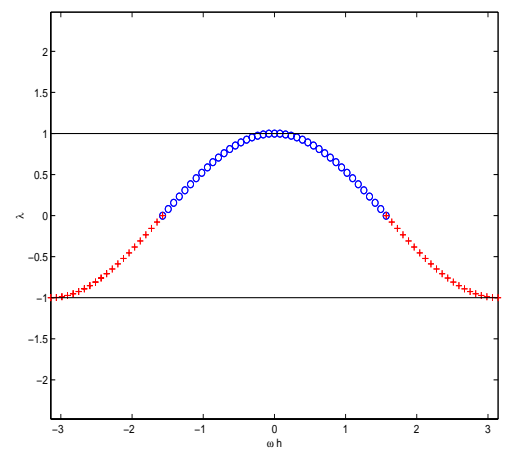

IP-DG $\mu=1 / h$

point-wise or cell-wise

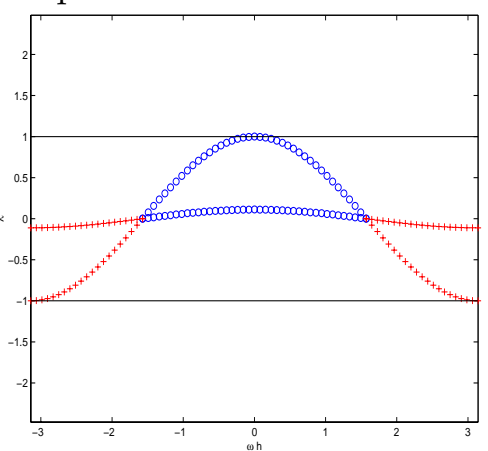

IP-DG $\mu=5 / h$

point-wise

$$
\circ: \omega_{\text {low }} \in[-\pi / 2 h, \pi / 2 h], \quad+: \omega_{\text {high }} \in[-\pi / h,-\pi / 2 h] \cup[\pi / 2 h, \pi / h]
$$

Figure 2: Eigenvalue spectra of $\widehat{M_{J O R}^{\mathrm{REL}}}(\omega)$.

In the Figures 2-4 we see that the Baumann and the symmetric DG method (both with $\mu=0$ ) show their instability by not damping the highest frequencies $|\omega| \approx \pi / h$. The high frequencies appear to be handled similarly as the low frequencies. We see that the IP-DG methods allow smoothing by the various relaxation methods, and that (the case $\mu=1 / h$ excluded) the point-wise relaxations are better than the cell-wise relaxations (high frequencies are better damped).

In Table 5 we summarize the damping of the high-frequencies and we show the corresponding optimal damping factors, $\alpha$, and smoothing factors for the damped relaxation methods in point-wise setting. We conclude that the pointwise block-relaxation methods are excellent smoothers. This brings us to focus more on their behavior in a multigrid algorithm in the next section. 


\begin{tabular}{|c|l|l|l|l|l|l|}
\hline $\mathrm{sf}=\max _{\omega}|\lambda(\omega)|$ & \multicolumn{2}{|c|}{ JOR } & \multicolumn{2}{c|}{ DGS } & \multicolumn{2}{c|}{ SDGS } \\
\cline { 2 - 7 }$\pi / 2 h \leq|\omega| \leq \pi / h$ & cell & point & cell & point & cell & point \\
\hline Baumann & - & 1.0 & 1.0 & 1.0 & 1.0 & 1.0 \\
\hline symmetric DG & - & 1.0 & 1.0 & 1.0 & 1.0 & 1.0 \\
\hline IP $\mu=1 / h$ & 1.0 & 1.0 & 0.447 & 0.447 & 0.200 & 0.200 \\
\hline IP $\mu=5 / h$ & 1.0 & 1.0 & 0.659 & 0.447 & 0.647 & 0.200 \\
\hline
\end{tabular}

\begin{tabular}{|c|l|l|l|l|l|l|}
\hline IP point-wise & $\alpha$ & sf & $\alpha$ & sf & $\alpha$ & sf \\
\hline$\mu=2 / h$ & 0.667 & 0.333 & 1.0 & 0.447 & 1.0 & 0.200 \\
\hline$\mu=5 / h$ & 0.667 & 0.333 & 1.0 & 0.447 & 1.0 & 0.200 \\
\hline \multicolumn{7}{c}{$\mathrm{sf}=\max _{\pi / 2 h \leq|\omega| \leq \pi / h}|\lambda(\omega)|$}
\end{tabular}

Table 5: Smoothing factors for the undamped (top) and the damped (bottom) relaxation methods. The damped relaxations are shown only for their point-wise ordered versions. and the damping factor is shown. 


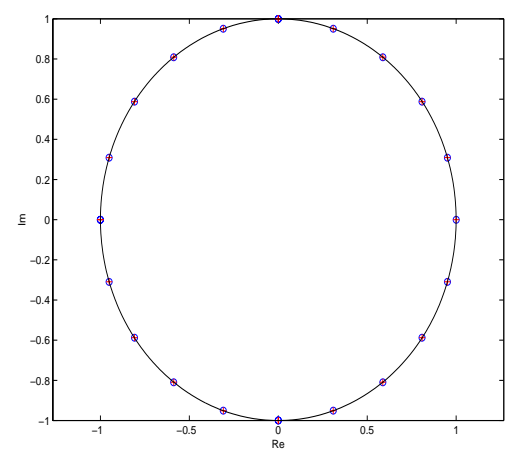

Baumann or symmetric-DG cell-wise

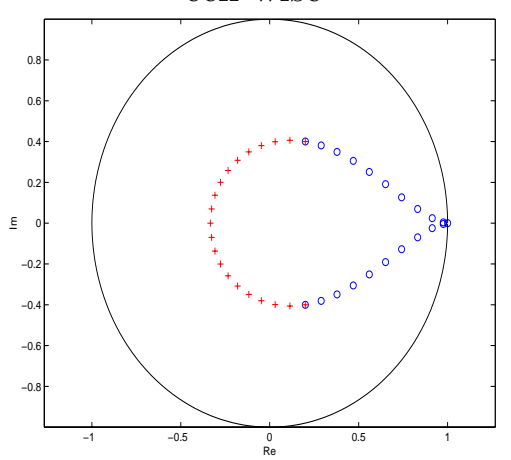

IP-DG $\mu=1 / h$

point-wise

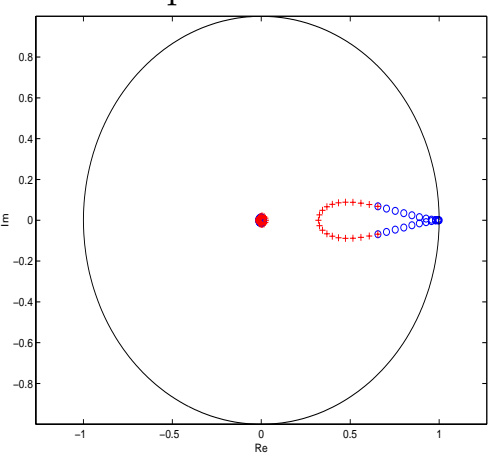

IP-DG $\mu=5 / h$ cell-wise

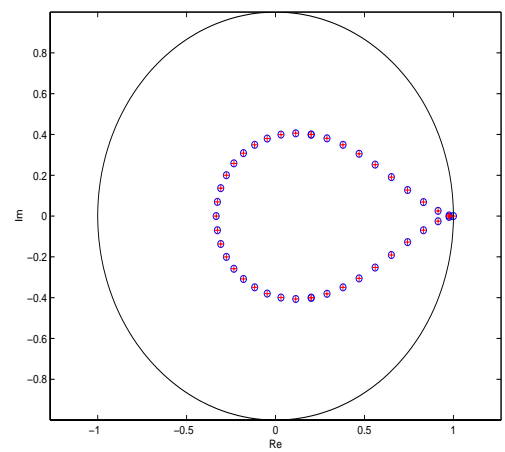

Baumann or symmetric-DG point-wise

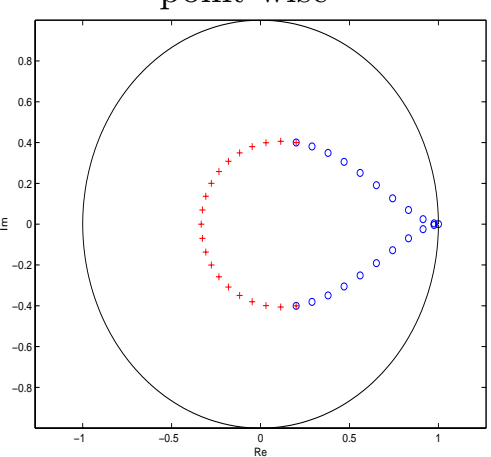

IP-DG $\mu=1 / h$ cell-wise

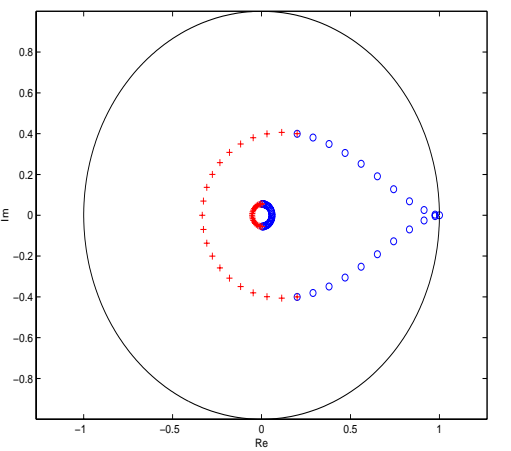

IP-DG $\mu=5 / h$ point-wise

$\circ: \omega_{\text {low }} \in[-\pi / 2 h, \pi / 2 h], \quad+: \omega_{\text {high }} \in[-\pi / h,-\pi / 2 h] \cup[\pi / 2 h, \pi / h]$

Figure 3: Eigenvalue spectra of $\widehat{M_{D G S}^{\mathrm{REL}}}(\omega)$, without damping $(\alpha=1)$ relative to unit circle. 


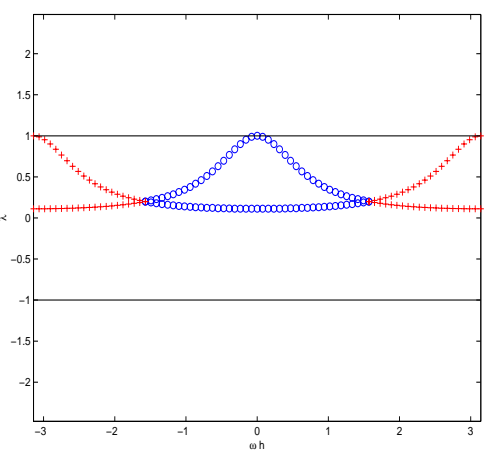

Baumann or symmetric-DG point-wise

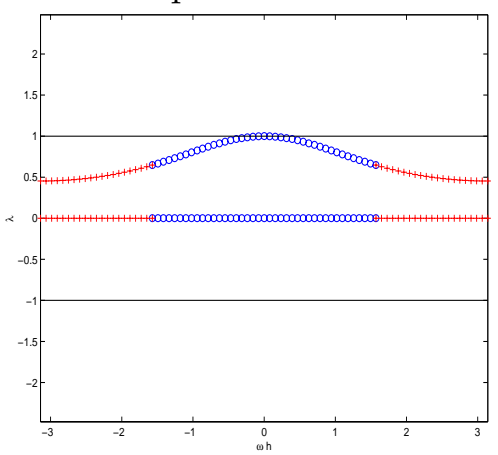

IP-DG $\mu=5 / h$ cell-wise

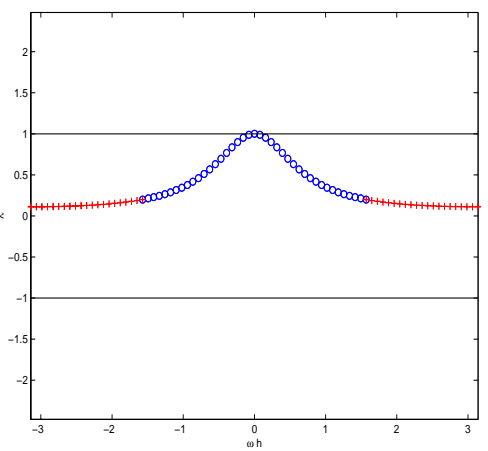

IP-DG $\mu=1 / h$ point- or cell-wise

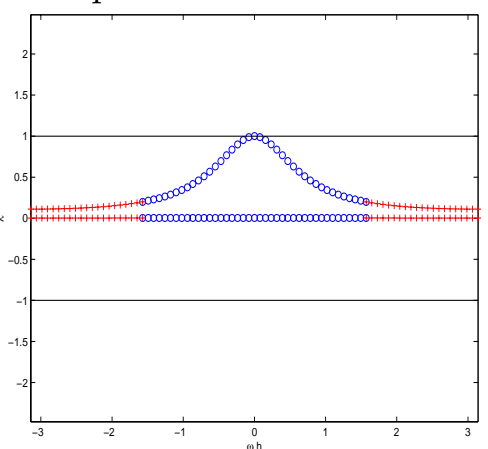

IP-DG $\mu=5 / h$ point-wise

$\circ: \omega_{\text {low }} \in[-\pi / 2 h, \pi / 2 h], \quad+: \omega_{\text {high }} \in[-\pi / h,-\pi / 2 h] \cup[\pi / 2 h, \pi / h]$

Figure 4: Eigenvalue spectra of $\widehat{M_{S G S}^{\mathrm{REL}}}(\omega)$. 


\subsection{The two-level analysis}

Now we study the two-level operator for the IP DG-method with three choices of $\mu$, viz. $\mu=1 / h, \mu=2 / h$ and $\mu=5 / h$, and we will compute optimal damping parameters for the smoothers JOR, DGS and SGS in combination with the coarse-grid correction, for the different choices of the parameter $\mu$. The amplification operator of the two-level algorithm for the error is given by

$$
\begin{aligned}
M_{h}^{T L A} & =\left(M_{h}^{\mathrm{REL}}\right)^{\nu_{2}} M_{h}^{\mathrm{CGC}}\left(M_{h}^{\mathrm{REL}}\right)^{\nu_{1}} \\
& =\left(M_{h}^{\mathrm{REL}}\right)^{\nu_{2}}\left(I-P_{h H} L_{H}^{-1} \bar{R}_{H h} L_{h}\right)\left(M_{h}^{\mathrm{REL}}\right)^{\nu_{1}},
\end{aligned}
$$

where $\nu_{1}$ and $\nu_{2}$ are the number of pre- (post-) relaxation sweeps respectively. $M_{h}^{C G C}$ is the amplification operator of the coarse grid correction. The amplification operator for the residue is

$$
\begin{aligned}
\bar{M}_{h}^{T L A} & =\left(\bar{M}_{h}^{R E L}\right)^{\nu_{2}} \bar{M}_{h}^{C G C}\left(\bar{M}_{h}^{R E L}\right)^{\nu_{1}} \\
& =\left(L_{h} M_{h}^{\mathrm{REL}} L_{h}^{-1}\right)^{\nu_{2}} L_{h} M_{h}^{\mathrm{CGC}} L_{h}^{-1}\left(L_{h} M_{h}^{\mathrm{REL}} L_{h}^{-1}\right)^{\nu_{1}} .
\end{aligned}
$$

It follows from [7] that the Fourier transform of the the coarse grid correction $M_{h}^{C G C}$ is

$$
\begin{gathered}
\widehat{M_{h}^{\mathrm{CGC}}}(\omega)=\left(\widehat{I_{h}}-\widehat{P_{h H}} \widehat{L_{H}^{-1}} \widehat{\bar{R}_{H h}} \widehat{L_{h}}\right)(\omega)=\left(\begin{array}{ll}
1 & 0 \\
0 & 1
\end{array}\right)- \\
\left(\begin{array}{c}
\widehat{P_{h}}(\omega) \\
\widehat{P_{h}}(\omega+\pi / h)
\end{array}\right)\left(\widehat{L_{H}}(\omega)\right)^{-1}\left(\begin{array}{ll}
\widehat{R_{h}}(\omega) & \widehat{\bar{R}_{h}}(\omega+\pi / h)
\end{array}\right)\left(\begin{array}{cc}
\widehat{L_{h}}(\omega) & 0 \\
0 & \widehat{L_{h}}(\omega+\pi / h)
\end{array}\right) .
\end{gathered}
$$

For our piecewise linear basis $\phi_{i, e}$, the interpolation $P_{h H}: S_{H} \rightarrow S_{h}$ so that $\left(P_{h H} u_{H}\right)(x)=$ $u_{H}(x)$ for all $x \in \mathbb{R} \backslash \mathbb{Z}_{h}$, is given by the stencil (for pointwise ordering):

$$
P_{h H} \approx\left[\begin{array}{ll|ll|ll}
0 & \frac{1}{2} & 1 & 0 & \frac{1}{2} & 0 \\
0 & \frac{1}{2} & 0 & 1 & \frac{1}{2} & 0
\end{array}\right]
$$

Because the DG discretization is of Galerkin type with equal test and trial space, the restriction of the residue, $\bar{R}_{H h}$, is the adjoint of the prolongation, $\bar{R}_{H h}=\left(P_{h H}\right)^{T}$. For the different penalty parameters $\mu$ and different smoothers JOR, DGS and SGS, the eigenvalue spectra of the two-level operator for the IP DG-method are computed from (3.3) and shown in the Figures 5-7.

We see that none of the methods converge for $\mu=1 / h$. However, for $\mu=2 / h$ or $\mu=2 / h$ all pointwise relaxations are excellent smoothers and we see fast convergence for the two-level algorithm.

Having found the spectra and having computed the largest and smallest real eigenvalue $\lambda_{\min }$ and $\lambda_{\max }$ we can determine the optimal damping parameter and the corresponding convergence rate for the damped relaxation method. The parameter, minimizing the spectral radius $\rho\left(M_{h}^{C G C} M_{h}^{R E L}\right)$ is given by:

$$
\alpha_{o p t}=\frac{2}{2-\left(\lambda_{\min }+\lambda_{\max }\right)},
$$

Seeing that the case $\nu=1$ will not show $h$-independent convergence, we show in the Tables 6 and 7 the damping parameters and the convergence rates for the cases $\nu=2$ and $\nu=5$. 


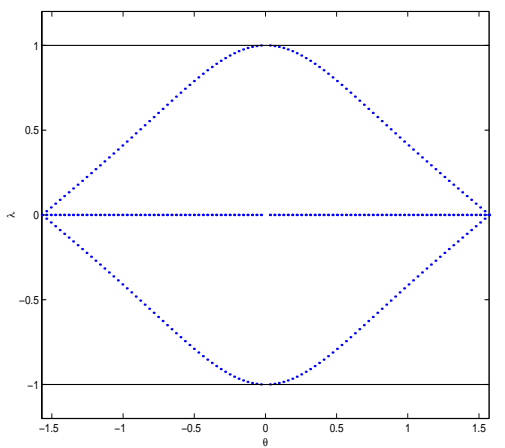

IP-DG $\mu=1 / h$

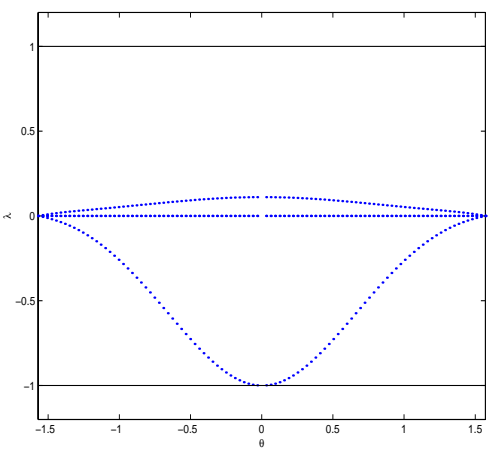

IP-DG $\mu=2 / h$

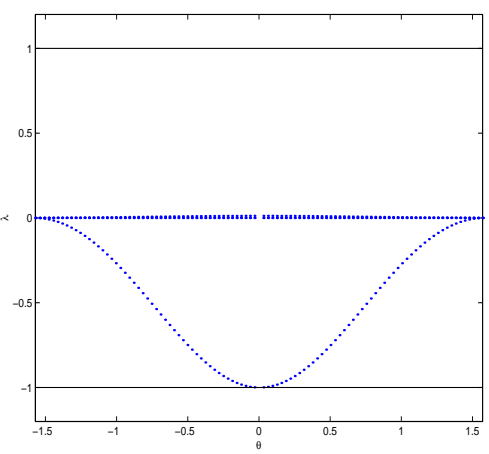

IP-DG $\mu=5 / h$

Figure 5: Eigenvalue spectra of $\mathbf{F T}\left(M_{h}^{C G C} M_{J O R}^{R E L}\right)(\omega)=\mathbf{F T}\left(\bar{M}_{h}^{C G C} \bar{M}_{J O R}^{R E L}\right)(\omega)$, without damping $(\alpha=1)$.

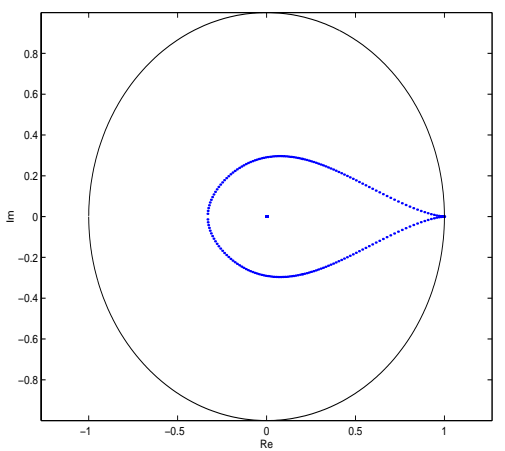

IP-DG $\mu=1 / h$

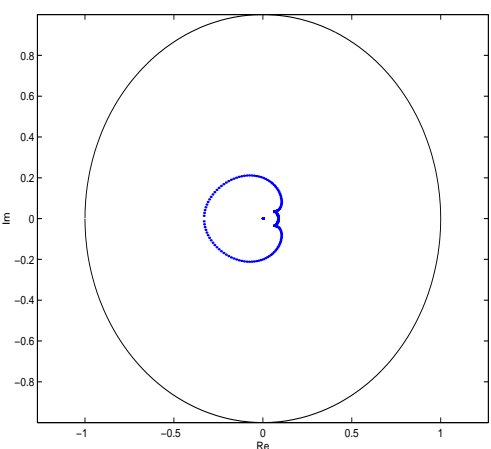

IP-DG $\mu=2 / h$

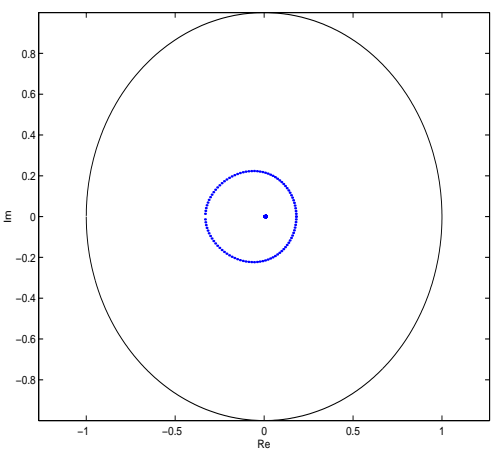

IP-DG $\mu=5 / h$

Figure 6: Eigenvalue spectra of $\mathbf{F T}\left(M_{h}^{C G C} M_{D G S}^{R E L}\right)(\omega)=\mathbf{F T}\left(\bar{M}_{h}^{C G C} \bar{M}_{D G S}^{R E L}\right)(\omega)$, without damping $(\alpha=1)$.

In order not only to know the asymptotic convergence rate but also the guaranteed converge behavior after one of two iteration sweeps, we also compute the spectral norms $\left\|M_{h}^{T L A}\right\|$, $\left\|\bar{M}_{h}^{T L A}\right\|,\left\|\left(\bar{M}_{h}^{T L A}\right)^{2}\right\|$. The results are shown in the Tables $8-10$. We see that the twolevel algorithm (and hence the multi-level algorithm) converges with a rate of about $0.2-$ 0.4 per iteration step and that reduction of the error and the residual is guaranteed, starting from the 2nd iteration step. 


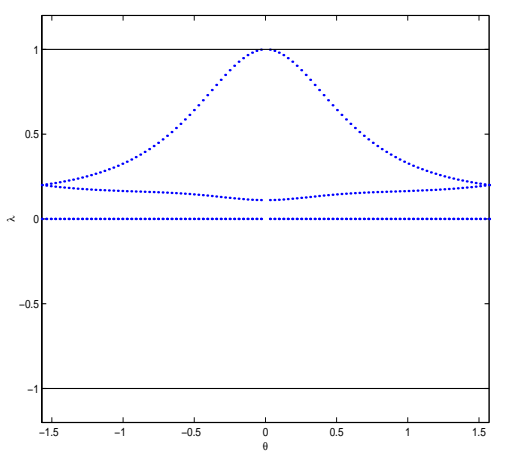

IP-DG $\mu=1 / h$

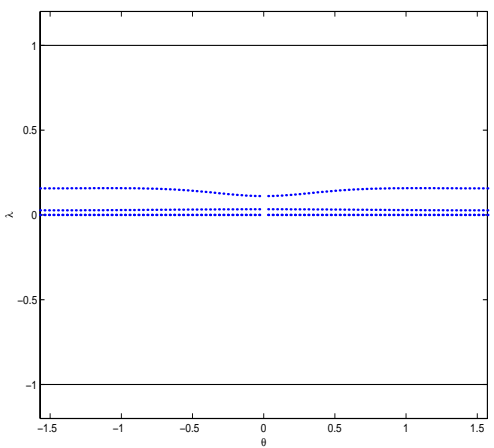

IP-DG $\mu=2 / h$

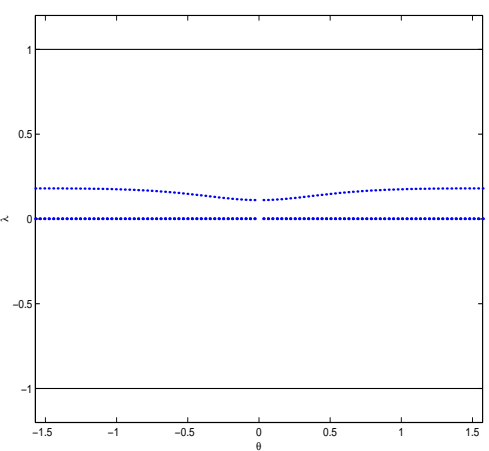

IP-DG $\mu=5 / h$

Figure 7: Eigenvalue spectra of two-level iteration with symmetric block-GS relaxation: $\mathbf{F T}\left(M_{D G S_{U}}^{R E L} M_{h}^{C G C} M_{D G S_{L}}^{R E L}\right)(\omega)=\mathbf{F T}\left(\bar{M}_{D G S_{U}}^{R E L} \bar{M}_{h}^{C G C} \bar{M}_{D G S_{L}}^{R E L}\right)(\omega)$, without damping $(\alpha=1)$.

\begin{tabular}{|c|c|c|}
\hline$\alpha_{o p t}$ & IP-DG $\mu=2 / h$ & IP-DG $\mu=5 / h$ \\
\hline$M_{h}^{C G C} M_{J O R}^{R E L}$ & 0.692 & 0.669 \\
\hline$M_{h}^{C G C} M_{D G S}^{R E L}$ & 0.897 & 0.928 \\
\hline
\end{tabular}

Table 6: Damping parameters for the two-level operators $\left(\rho\left(M_{h}^{C G C} M_{h}^{R E L}\right)=\right.$ $\left.\rho\left(\bar{M}_{h}^{R E L} \bar{M}_{h}^{C G C}\right)\right)$.

\section{NUMERICAL RESUlts}

In this section we check by numerical experiments the spectral radii of the two-level operators with damped Jacobi-, $M_{h}^{C G C} M_{J O R}^{R E L}$, Gauss-Seidel-, $M_{h}^{C G C} M_{D G S}^{R E L}$, and symmetric GaussSeidel relaxation, $M_{D G S_{U}}^{R E L} M_{h}^{C G C} M_{D G S_{L}}^{R E L}$, for the IP-DG method with the penalty parameters $\mu=2 / h$ and $\mu=5 / h$. For that purpose we consider an inhomogeneous Poisson equation,

$$
-u_{x x}=\frac{e^{x / \epsilon}}{\epsilon^{2}\left(\epsilon^{1 / \epsilon}-1\right)}, \quad \text { with } u(0)=0, u(1)=0, \epsilon=1 / 64,
$$

which has a sharp boundary layer type solution. For the discrete system we use the linear basis polynomials and we set the meshwidth to $h=2^{-6}$. Our initial approximation is the grid-function $u_{h}^{0}=u_{h, \mathrm{PRE}}^{0}=\sin (1 / 2 \pi j)$. We apply a pre-relaxation sweep

$$
u_{h, \mathrm{PRE}}^{i+1}=u_{h, \mathrm{PRE}}^{i}+B_{h}\left(f_{h}-L_{h} u_{h, \mathrm{PRE}}^{i}\right),
$$

\begin{tabular}{|c|c|c|}
\hline$\rho\left(M_{h}^{C G C} M_{h}^{R E L}\right)$ & IP-DG $\mu=2 / h$ & IP-DG $\mu=5 / h$ \\
\hline$M_{h}^{C G C} M_{J O R}^{R E L}$ & 0.385 & 0.339 \\
\hline$M_{h}^{C G C} M_{D G S}^{R E L}$ & 0.217 & 0.238 \\
\hline$M_{D G S_{U}}^{R E L} M_{h}^{C G C} M_{D G S_{L}}^{R E L}$ & 0.156 & 0.180 \\
\hline
\end{tabular}

Table 7: Spectral radii $\rho\left(M_{h}^{C G C} M_{h}^{R E L}\right)=\rho\left(\bar{M}_{h}^{R E L} \bar{M}_{h}^{C G C}\right)$ for damping parameters in Table 6. 


\begin{tabular}{|l|c|c|c|}
\hline & $M_{h}^{C G C} M_{J O R}^{R E L}$ & $M_{h}^{C G C} M_{D G S}^{R E L}$ & $M_{D G S_{U}}^{R E L} M_{h}^{C G C} M_{D G S_{L}}^{R E L}$ \\
\hline IP-DG $(\mu=2 / h)$ & 0.543 & 0.392 & 0.207 \\
\hline IP-DG $(\mu=5 / h)$ & 0.478 & 0.417 & 0.250 \\
\hline
\end{tabular}

Table 8: The spectral norm $\left(\sigma_{\max }\right)$ after 1 iteration for the error with optimal damping.

\begin{tabular}{|l|c|c|c|}
\hline & $\bar{M}_{h}^{C G C} \bar{M}_{J O R}^{R E L}$ & $\bar{M}_{h}^{C G C} \bar{M}_{D G S}^{R E L}$ & $\bar{M}_{D G S_{U}}^{R E L} \bar{M}_{h}^{C G C} \bar{M}_{D G S_{L}}^{R E L}$ \\
\hline IP-DG $(\mu=2 / h)$ & 1.071 & 1.019 & 0.340 \\
\hline IP-DG $(\mu=5 / h)$ & 1.056 & 1.028 & 0.343 \\
\hline
\end{tabular}

Table 9: The spectral norm $\left(\sigma_{\max }\right)$ after 1 iteration for the residue with optimal damping.

with $B_{h}$ the approximate inverse of $L_{h}$ as given in Table 3, and the coarse grid correction

$$
u_{h, \mathrm{POST}}^{0}=u_{h, \mathrm{PRE}}^{\nu_{1}}+P_{h H} L_{H}^{-1} \bar{R}_{H h}\left(f_{h}-L_{h} u_{h, \mathrm{PRE}}^{\nu_{1}}\right) .
$$

In case of symmetric damped Gauss-Seidel we apply an additional post relaxation sweep

$$
u_{h, \mathrm{POST}}^{i+1}=u_{h, \mathrm{POST}}^{i}+B_{h}^{T}\left(f_{h}-L_{h} u_{h, \mathrm{POST}}^{i}\right) .
$$

To be consistent with the Fourier analysis we measure the residue in the 2-norm

$$
\left\|d_{h}\right\|_{2}=\left\|f_{h}-L_{h} u_{h}\right\|_{2}=\left(\sum_{e=1}^{64} \sum_{j=1}^{2} d_{h e, j}^{2}\right)^{\frac{1}{2}}
$$

The convergence of the residue is shown in Figure 8. The convergence factors as observed, are given in Table 11.

Both for $\mu=2 / h$ and for $\mu=5 / h$ we see convergence, starting from the first iteration sweep. Furthermore, for the IP-DG method with $\mu=5 / h$ the observed convergence factors correspond very well with spectral radii shown in Table 7. Only for the IP-DG method with $\mu=2 / h$ the spectral radii of the Fourier analysis seem too optimistic compared with the convergence factors in Table 11. This is clearly caused by a boundary effect (as can be seen if we study the slowest converging component, which is exponentially growing towards the boundary). This is related to the fact that, as $\mu$ approaches $1 / h$, the two-level algorithm becomes singular. This singularity effect disappears for larger values of $\nu=h \mu$.

\begin{tabular}{|l|c|c|c|}
\hline & $\bar{M}_{h}^{C G C} \bar{M}_{J O R}^{R E L}$ & $\bar{M}_{h}^{C G C} \bar{M}_{D G S}^{R E L}$ & $\bar{M}_{D G S_{U}}^{R E L} \bar{M}_{h}^{C G C} \bar{M}_{D G S_{L}}^{R E L}$ \\
\hline IP-DG $(\mu=2 / h)$ & 0.411 & 0.200 & 0.030 \\
\hline IP-DG $(\mu=5 / h)$ & 0.357 & 0.244 & 0.035 \\
\hline
\end{tabular}

Table 10: The spectral norm $\left(\sigma_{\max }\right)$ after 2 iterations for the residue with optimal damping. 


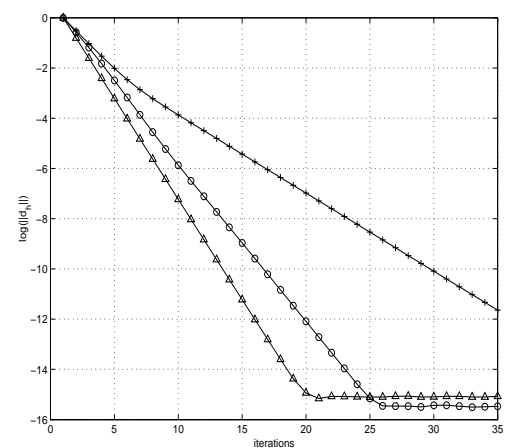

IP-DG $m u=2 / h$

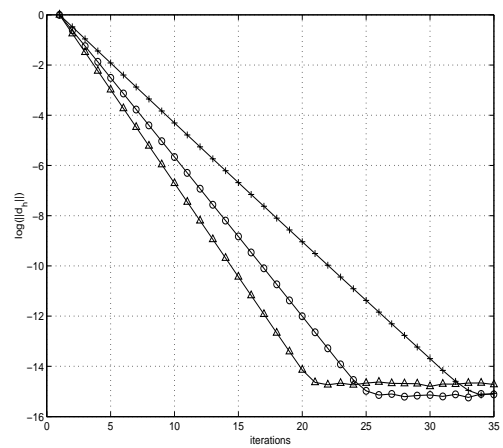

IP-DG $\mu=5 / h$

+: JOR; o: DGS; $\triangle$ : SGS.

Figure 8: $\log \left(\left\|d_{h}\right\|_{2}\right)$ as function of iterations for the two-level iteration operator on the error

\begin{tabular}{|c|c|c|}
\hline$\rho\left(M_{h}^{C G C} M_{h}^{R E L}\right)$ & IP-DG $\mu=2 / h$ & IP-DG $\mu=5 / h$ \\
\hline$M_{h}^{C G C} M_{J O R}^{R E L}$ & 0.48 & 0.34 \\
\hline$M_{h}^{C G C} M_{D G S}^{R E L}$ & 0.24 & 0.23 \\
\hline$M_{D G S_{U}}^{R E L} M_{h}^{C G C} M_{D G S_{L}}^{R E L}$ & 0.17 & 0.18 \\
\hline
\end{tabular}

Table 11: Numerically obtained convergence factors corresponding with $\rho\left(M_{h}^{C G C} M_{h}^{R E L}\right)=$ $\rho\left(\bar{M}_{h}^{R E L} \bar{M}_{h}^{C G C}\right)$ for damping parameters as in Table 6 .

\section{Conclusion}

In an earlier paper we have shown that multigrid iteration can be quite efficient for the solution of elliptic equations that are discretized by higher-order discontinuous Galerkin discretization, provided that a block (Jacobi or Gauss-Seidel) relaxation is used, based on a pointwise (instead of a cell-wise) ordering.

In this paper we have studied the solution of the discrete equations for the discontinuous Galerkin method with piecewise linear test- and trial functions. It is well-known $[1,10]$ that in this case the DG method requires an interior penalty (IP) parameter $\mu>1$ in order to guarantee that the discrete equations are stable.

We show that in this case, again, a multigrid method can be used to solve the corresponding discrete equations if block relaxation is used, based on the pointwise ordering. If a suitable IP parameter $\mu>1$ is chosen, the block Jacobi or (symmetric) block Gauss-Seidel relaxation have a good smoothing property.

Using Fourier analysis, in this paper, for feasible $\mu$-values, we compute optimal damping parameters for the relaxation methods and the corresponding two-level convergence rates. In view of the hierarchical structure of the DG multigrid-algorithm proposed in [7], the present results can be used to justify the local use of -also- a low order discretization in the hierarchical scale of methods in the neighborhood of singularities, or in order to accelerate the solution of the higher-order discretization. 


\section{REFERENCES}

1. D.N. Arnold. An interior penalty finite element method with discontinuous elements. SIAM J.Numer.Anal., 19:742, 1982.

2. D.N. Arnold, F. Brezzi, B. Cockburn, and D. Marini. Unified analysis of discontinuous Galerkin methods for elliptic problems. SIAM J Numer. Anal., pages 1749 - 1779, 2002.

3. I. Babuška, C.E. Baumann, and J.T. Oden. A discontinuous finite element method for diffusion problems: 1-d analysis. Computers Math. Applic., page to appear, 1999.

4. C. E. Baumann and J. T. Oden. An hp-adaptive discontinuous finite element method for computational fluid dynamics. The university of Texas at Austin, 1997.

5. B. Cockburn. Discontinuous Galerkin methods for convection dominated problems, 1999. Lecture Notes, available at http://www. math. umn. edu/ cockburn/LectureNotes.html.

6. B. Cockburn and C.W. Shu. The local finite element method for convection-diffusion systems. Siam J. Numer. Anal., 35:2440-2463, 1998.

7. P.W. Hemker, W. Hoffmann, and M.H. van Raalte. Two-level fourier analysis of a multigrid approach for discontinuous galerkin discretisation. Technical report, CWI, 2002. submitted to SIAM SISC.

8. P. Houston, C. Schwab, and E. Süli. Discontinuous $h p$-finite element methods for advection-diffusion problems. Technical Report No. 2000-07, ETHZ, Zürich, Switzerland, 2000.

9. J.T. Oden, I. Babuška, and C.E. Baumann. A discontinuous $h p$ finite element method for diffusion problems. J. Comp. Phys., 146:491-519, 1998.

10. B. Rivière, M. F. Wheeler, and V. Girault. Improved energy estimates for interior penalty, cpnstrained and dicontinuous galerkin methods for elliptic problems. Part I. Computational Geosciences, 3:337-360, 1999.

11. E. Süli, P. Houston, and C. Schwab. $h p$-finite element methods for hyperbolic problems. Technical Report No. 99-14, ETHZ, Zürich, Switzerland, 1999.

12. E. Süli, C. Schwab, and P. Houston. hp-dgfem for partial differential equations with nonnegative characteristic form. Technical Report No. 99-13, ETHZ, Zürich, Switzerland, 1999.

13. M.F. Wheeler. An elliptic collocation-finite element method with interior penalties. SIAM J.Numer.Anal., 15:152, 1978. 